University of South Carolina

Scholar Commons

$9-2010$

\title{
Muscular and Performance Fitness and the Incidence of Type 2 Diabetes: Prospective Study of Japanese Men
}

\author{
Susumu S. Sawada
}

I-Min Lee

Hisashi Naito

Koji Tsukamoto

Takashi Muto

See next page for additional authors

Follow this and additional works at: https://scholarcommons.sc.edu/

sph_epidemiology_biostatistics_facpub

Part of the Public Health Commons

\section{Publication Info}

Published in Journal of Physical Activity and Health, Volume 7, Issue 5, 2010, pages 627-632.

Sawada, S. S., Lee, I-M., Naito, H., Tsukamoto, K., Muto, T., \& Blair, S. N. (2010). Muscular and performance fitness and the incidence of type 2 diabetes: Prospective study of Japanese men. Journal of Physical Activity and Health, 7(5), 627-632.

(c) Journal of Physical Activity and Health, 2010, Human Kinetics

This Article is brought to you by the Epidemiology and Biostatistics at Scholar Commons. It has been accepted for inclusion in Faculty Publications by an authorized administrator of Scholar Commons. For more information, please contact digres@mailbox.sc.edu. 


\section{Author(s)}

Susumu S. Sawada, I-Min Lee, Hisashi Naito, Koji Tsukamoto, Takashi Muto, and Steven N. Blair 


\title{
Muscular and Performance Fitness and the Incidence of Type 2 Diabetes: Prospective Study of Japanese Men
}

\author{
Susumu S. Sawada, I-Min Lee, Hisashi Naito, Koji Tsukamoto, Takashi Muto, \\ and Steven N. Blair
}

\begin{abstract}
Background: Limited data are available on the relationship between muscular and performance fitness (MPF) and the incidence of type 2 diabetes. Methods: A cohort of 3792 Japanese men completed a medical examination that included MPF and cardiorespiratory fitness tests. MPF index composite score was calculated using Z-scores from vertical jump, sit-ups, side step, and functional reach tests. Results: The mean follow-up period was 187 months (15.6 years). There were 240 patients who developed type 2 diabetes during follow-up. Relative risks and $95 \%$ confidence intervals (CI) for incidence of diabetes across baseline quartiles of MPF index composite score were obtained using the Cox proportional hazards model while adjusting for age, BMI, diastolic blood pressure, cigarette smoking, alcohol intake, and family history of diabetes. The relative risks for developing diabetes across quartiles of MPF index composite scores (lowest to highest) were 1.0 (referent), 1.15 (95\% CI 0.83-1.60), $1.10(0.78-1.55)$, and $0.57(0.37-0.90)(P$ for trend $=.061)$. These results were attenuated after adjustment for cardiorespiratory fitness $(P$ for trend $=.125)$. Conclusions: This prospective study suggests that MPF is a predictor of type 2 diabetes, although its predictive ability was attenuated after adjusting for cardiorespiratory fitness.
\end{abstract}

Keywords: cardiorespiratory fitness, maximal oxygen uptake, epidemiology

It is well established that physical activity is an important independent predictor of type 2 diabetes. ${ }^{1,2}$ Physical activity improves glucose levels, reduces adiposity, increases muscle mass and the glucose-transporter 4 in muscle tissues, and affects the reduction of insulin resistance. ${ }^{3}$ Therefore, physical activity may prevent or delay type 2 diabetes.

Although there is a genetic component, cardiorespiratory fitness is primarily determined by physical activity habits. ${ }^{4}$ Thus, cardiorespiratory fitness can be an objective marker for physical activity. Haskell et al reported that a major health-related physical fitness component is cardiorespiratory fitness. ${ }^{5}$ Several prospective studies reported that cardiorespiratory fitness is a strong independent predictor of type 2 diabetes. ${ }^{6-8}$ However, physical activity does not only affect cardiorespiratory fitness, it also has an effect on other fitness components. Therefore, other fitness components may be independent predictors for type 2 diabetes. However, to our knowledge, only 1 prospective cohort study has examined the relationship

Sawada and Tsukamoto are with the Health Promotion Center, Tokyo Gas Co., Tokyo, Japan. Lee is with the Dept of Epidemiology, Harvard School of Public Health, Boston, MA. Naito is with the Dept of Exercise Physiology, Juntendo University, Tokyo, Japan. Muto is with the Dept of Public Health, Dokkyo Medical University School of Medicine, Tochigi, Japan. Blair is with the Dept of Exercise Science, University of South Carolina, Columbia, SC. between muscular and performance components of physical fitness and the incidence of type 2 diabetes. ${ }^{9}$ Thus, to provide more information, we conducted a prospective study of Japanese male workers, to determine whether muscular and performance fitness (MPF) is an independent predictor for type 2 diabetes.

\section{Methods}

\section{Subjects}

All subjects were employees of the Tokyo Gas Company that supplies natural gas to the Tokyo area. At baseline, the subjects who were selected did not have diabetes, cardiovascular disease, hypertension, or gastrointestinal disease; and completed MPF and cardiorespiratory fitness tests, annual health examinations, and questionnaires regarding their health habits in 1983. Female workers were excluded due to their small numbers. Eligible subjects consisted of 3792 Japanese male workers between 18 and 38 years of age. All the subjects were followed until the first of the following events: development of type 2 diabetes $(n=240)$, death $(n=42)$, retirement ( $n$ $=88$ ) or completion of the study in 1999 .

\section{Clinical Examinations}

The Industrial Safety and Health Law in Japan requires the employer to conduct annual clinical examinations of all employees. Employees are required by law to 
participate. Thus, all the subjects were given a clinical examination annually. BMI was calculated from measured weight and height. Resting blood pressure was obtained with standard auscultatory methods. A selfadministered questionnaire was conducted before the start of the fitness tests to assess the individual's smoking habit, alcohol habit, and a family history of diabetes.

\section{Fitness Tests}

We used the vertical jump test as an indicator of lower extremity muscular power. In this test, the subject stood with his side to a wall and reached up with the hand closest to the wall. Keeping the feet flat on the ground, the point of the fingertips was marked and recorded. The subject then stood away from the wall, and jumped vertically as high as possible while attempting to touch the wall at the highest point of the jump. We used the sit-up test as an indicator of muscular endurance of the abdominal muscles. Sit-ups were performed from the supine position, with knees flexed at a 90-degree angle. One complete sit-up entailed curling the trunk from the supine position to touching the elbows to the knees and returning to the start position; the number completed in 30 seconds was counted. We used the side step test as an indicator of agility. The subject stood at a center line, then jumped $100 \mathrm{~cm}$ to the side and touched a line with the closest foot, jumped back to the center, then jumped $100 \mathrm{~cm}$ to the other side and back to the center again for 1 complete cycle. The number of cycles completed in 20 seconds was counted. We used the functional reach test as an indicator of trunk flexibility. This was performed from the standing position on a measurement box with a scale. The subject reached toward the floor, with knees straight, and the furthest point of reach was recorded. Z-scores were calculated for all MPF tests. Z-scores of each test were summed to construct MPF index composite score. Subjects also underwent a submaximal exercise test on a cycle ergometer to assess cardiorespiratory fitness. The maximal oxygen uptake $\left(\mathrm{VO}_{2 \max }\right)$, an index of cardiorespiratory fitness, was estimated using, the Åstrand-Ryming Nomogram ${ }^{10}$ and the Nomogram correction factors. ${ }^{11}$ Additional details of the cardiorespiratory fitness test have previously been reported. ${ }^{7}$

\section{Diagnosis of Type 2 Diabetes}

The development of type 2 diabetes was based on one of the following 3 diagnostic parameters: (1) plasma glucose levels exceeded more than $11.1 \mathrm{mmol} \cdot \mathrm{L}^{-1}\left(200 \mathrm{mg} \cdot \mathrm{dL}^{-1}\right)$ 2 hours after an oral glucose tolerance test, conducted in men with urinary glucose detected at a follow-up annual health examination, (2) subjects themselves reported current therapy with hypoglycemic medication (insulin or oral hypoglycemic agent) when they were interviewed at the annual clinical examination, or (3) using fasting plasma glucose tests which had been adopted since 1988 . The criterion for fasting plasma glucose level for the diagnosis of type 2 diabetes was more than $7.0 \mathrm{mmol} \cdot \mathrm{L}^{-1}$ $\left(126 \mathrm{mg} \cdot \mathrm{dL}^{-1}\right) .^{12}$

\section{Data Analysis}

We first compared characteristics of subjects according to fourths of MPF index composite scores. The KruskalWallis test was used for categorical variables, while differences in the means of continuous measurements were tested by analysis of variance.

Cox proportional hazards model was used to study the relationship between MPF and the incidence of type 2 diabetes, adjusted for age (years), BMI $\left(\mathrm{kg} \cdot \mathrm{m}^{-2}\right)$, diastolic blood pressure $(\mathrm{mmHg})$, family history of diabetes (present or not), smoking status (never-smokers, smokers who quit over 1 year ago, smokers who quit within 1 year, smokers of 1 to 20 cigarettes per day, 21 to 40 cigarettes per day, 41 or more cigarettes per day), and alcohol intake (none, 1-22g per day, 23-44g per day, 45-66g per day, $67 \mathrm{~g}$ or more per day). Systolic and diastolic blood pressure were highly correlated; thus, we only adjusted for diastolic blood pressure to avoid multicollinearity. Relative risks for incidence of type 2 diabetes and $95 \%$ confidence intervals (CI) were obtained by using the group with the lowest MPF as the reference category. The proportionality assumption of the model was tested using a log-minus-log plot; no evidence of violation was found.

All P values were 2-sided. Analyses were performed using SPSS, version 11.0J (SPSS, Inc., Chicago, IL).

\section{Results}

The mean follow-up period was 187 months (15.6 years), and the total follow-up experience for the cohort was 59,089 man-years of observation. There were 240 patients who developed type 2 diabetes during follow-up. 93 patients $(39 \%)$ were ascertained using oral glucose tolerance tests, 94 patients $(39 \%)$ by fasting plasma glucose test, and 53 patients $(22 \%)$ reported current therapy for diabetes at the annual clinical examination. The average age of subjects was 29.6 years (range: 18 to $38 \mathrm{yr}$ ) at baseline.

Table 1 shows baseline characteristics of individuals according to MPF levels. Men in the higher MPF groups were younger in age than those in the lower groups; they also had higher estimated $\mathrm{VO}_{2 \max }$ levels. Men in the highest MPF group had the lowest levels of BMI and diastolic blood pressure.

Table 2 shows the Pearson correlation coefficients among each of the components of MPF and cardiorespiratory fitness. The correlations tended to be low to moderate in magnitude. Vertical jump and sit-ups, vertical jump and side step, and sit-ups and side-step had the highest correlations ( $r=.37, .39$, and .42 respectively). Each of MPF tests and cardiorespiratory fitness were weakly correlated. MPF index composite score had a positive correlation with cardiorespiratory fitness $(r=.24)$. 
Table 1 Baseline Characteristics of Men According to Muscular and Performance Fitness (MPF) Levels

\begin{tabular}{lcccccc}
\hline Characteristics & All men & $\mathbf{Q}_{1}$ (lowest) & $\mathbf{Q}_{2}$ & $\mathbf{Q}_{3}$ & $\mathbf{Q}_{4}$ (highest) & $\boldsymbol{P}$ value \\
\hline $\mathrm{N}$ & 3792 & 948 & 948 & 948 & 948 & \\
Age (years) & $29.6 \pm 4.7$ & $31.9 \pm 3.9$ & $30.4 \pm 4.4$ & $28.9 \pm 4.6$ & $27.2 \pm 4.5$ & $<.001 *$ \\
Estimated $\mathrm{VO}_{2 \max }\left(\mathrm{mL} \cdot \mathrm{kg}^{-1} \cdot \mathrm{min}^{-1}\right)$ & $40.4 \pm 7.4$ & $38.6 \pm 7.0$ & $39.2 \pm 6.7$ & $40.8 \pm 7.3$ & $43.2 \pm 7.7$ & $<.001 *$ \\
$\mathrm{BMI}\left(\mathrm{kg} \cdot \mathrm{m}^{-2}\right)$ & $22.9 \pm 2.5$ & $23.2 \pm 2.7$ & $23.1 \pm 2.5$ & $22.9 \pm 2.5$ & $22.5 \pm 2.2$ & $<.001 *$ \\
Systolic blood pressure $(\mathrm{mmHg})$ & $125.5 \pm 11.6$ & $125.6 \pm 12.1$ & $125.8 \pm 11.6$ & $125.5 \pm 11.6$ & $125.0 \pm 11.1$ & $.520 *$ \\
Diastolic blood pressure $(\mathrm{mmHg})$ & $72.7 \pm 9.0$ & $74.1 \pm 8.9$ & $73.1 \pm 8.9$ & $72.5 \pm 9.0$ & $71.1 \pm 8.8$ & $<.001 *$ \\
Family history of diabetes $(\%)$ & 22.8 & 24.4 & 21.9 & 22.9 & 21.8 & $.527 \dagger$ \\
Current smokers $(\%)$ & 67.8 & 68.1 & 68.3 & 68.3 & 66.6 & $.860 \dagger$ \\
Current drinkers $(\%)$ & 70.3 & 72.9 & 70.7 & 70.3 & 67.2 & $.058 \dagger$ \\
Vertical jump $(\mathrm{cm})$ & $56.1 \pm 7.1$ & $50.0 \pm 5.6$ & $54.4 \pm 5.1$ & $57.5 \pm 5.2$ & $62.6 \pm 5.7$ & $<.001 *$ \\
Sit-ups $\left(\mathrm{n} \cdot 30\right.$ sec $\left.^{-1}\right)$ & $23.9 \pm 4.1$ & $20.2 \pm 3.5$ & $23.0 \pm 2.7$ & $24.9 \pm 2.8$ & $27.7 \pm 3.1$ & $<.001 *$ \\
Side step $\left(\mathrm{n} \cdot 20 \mathrm{sec}^{-1}\right)$ & $49.0 \pm 4.8$ & $44.5 \pm 4.0$ & $47.9 \pm 3.3$ & $50.0 \pm 3.4$ & $53.4 \pm 3.7$ & $<.001 *$ \\
Functional reach $(\mathrm{cm})$ & $13.5 \pm 5.9$ & $9.4 \pm 5.7$ & $12.4 \pm 5.0$ & $14.8 \pm 5.1$ & $17.4 \pm 4.7$ & $<.001 *$ \\
MPF index composite score + & $0.00 \pm 2.69$ & $-3.43 \pm 1.38$ & $-0.86 \pm 0.50$ & $0.87 \pm 0.53$ & $3.41 \pm 1.30$ & $<.001 *$ \\
\hline
\end{tabular}

Note. Data are means $\pm \mathrm{SD}$, unless otherwise specified.

* from ANOVA.

$\dagger$ from Kruskal-Wallis test.

$\ddagger$ Sum of Z-scores.

Table 2 Pearson Correlation Coefficients Among Each Muscular and Performance Fitness Component and Cardiorespiratory Fitness

\begin{tabular}{lcccc}
\hline & Vertical jump & Sit-ups & Side step & Functional reach \\
\hline Sit-ups & .37 & - & - & - \\
Side step & .39 & .42 & - & - \\
Functional reach & .14 & .18 & .12 & - \\
Cardiorespiratory fitness & .18 & .20 & .19 & .08 \\
\hline
\end{tabular}

Note. All correlations statistically significantly at $P<.001$.

Table 3 shows the relative risks for type 2 diabetes by MPF index composite scores, fitness levels for each muscular and performance test, and cardiorespiratory fitness levels, with the lowest level used as the referent. There was an inverse dose-response trend across MPF index composite scores for type 2 diabetes. After adjusting for BMI, diastolic blood pressure, family history of diabetes, smoking status, and alcohol intake, the group with the highest MPF had a 43\% lower risk of type 2 diabetes than the group with the lowest MPF. After further adjustment for cardiorespiratory fitness, the relationship was diminished $(P$ for trend $=.125)$. These analyses were repeated, excluding subjects whose BMI was over 25 (n =731),${ }^{13}$ because excess abdominal fat can be a physical limitation for sit-ups or functional reach. The analyses were also repeated, excluding individuals who developed type 2 diabetes within 5 years $(n=12)$. The results were not substantially changed with these exclusions (data not shown). Men in the higher sit-up score groups all had significantly lower relative risk for type 2 diabetes than men in the lowest sit-up score group. In addition, higher side step and functional reach score groups had lower risks of type 2 diabetes; however, is the results were not significant. Conversely, the higher vertical jump score groups had higher relative risks. Previous prospective studies have shown a strong inverse relationship between cardiorespiratory fitness and the incidence of type 2 diabetes. ${ }^{6-8}$ We also observed a strong inverse association between cardiorespiratory fitness and type 2 diabetes, after adjustment for MPF ( $P$ for trend <.001).

Finally, we calculated multivariate relative risks for incidence of type 2 diabetes according to groups of men 
Table 3 Relative Risks of Incidence of Type 2 Diabetes, According to Muscular and Performance Fitness (MPF) Index Composite Scores, Each MPF Levels, and Cardiorespiratory Fitness Levels

\begin{tabular}{|c|c|c|c|c|c|}
\hline & $Q_{1}$ (lowest) & $Q_{2}$ & $Q_{3}$ & $Q_{4}$ (highest) & $P$ for trend \\
\hline \multicolumn{6}{|l|}{ MPF index composite score } \\
\hline $\mathrm{n}$ & 948 & 948 & 948 & 948 & \\
\hline Man-years of follow-up & 14,638 & 14,709 & 14,797 & 14,945 & \\
\hline No. of cases & 77 & 72 & 63 & 28 & \\
\hline Age-adjusted RR (95\% CI) & 1.00 & $1.02(0.74-1.41)$ & $0.96(0.68-1.36)$ & $0.47(0.30-0.74)$ & .005 \\
\hline Multivariate RR* $(95 \% \mathrm{CI})$ & 1.00 & $1.15(0.83-1.60)$ & $1.10(0.78-1.55)$ & $0.57(0.37-0.90)$ & .061 \\
\hline Multivariate $\mathrm{RR} \dagger(95 \% \mathrm{CI})$ & 1.00 & $1.14(0.82-1.58)$ & $1.09(0.78-1.54)$ & $0.62(0.39-0.97)$ & .125 \\
\hline \multicolumn{6}{|l|}{ Vertical jump } \\
\hline Multivariate $\mathrm{RR} \ddagger(95 \% \mathrm{CI})$ & 1.00 & $1.04(0.73-1.50)$ & $1.40(0.99-1.99)$ & $1.35(0.90-2.01)$ & .052 \\
\hline \multicolumn{6}{|l|}{ Sit-ups } \\
\hline Multivariate $\mathrm{RR} \ddagger(95 \% \mathrm{CI})$ & 1.00 & $0.64(0.45-0.93)$ & $0.60(0.42-0.84)$ & $0.61(0.41-0.92)$ & .004 \\
\hline \multicolumn{6}{|l|}{ Side step } \\
\hline Multivariate $\mathrm{RR} \ddagger(95 \% \mathrm{CI})$ & 1.00 & $0.86(0.60-1.23)$ & $1.00(0.70-1.41)$ & $0.83(0.53-1.29)$ & .619 \\
\hline \multicolumn{6}{|l|}{ Functional reach } \\
\hline Multivariate $\mathrm{RR} \ddagger(95 \% \mathrm{CI})$ & 1.00 & $0.80(0.56-1.13)$ & $0.85(0.59-1.22)$ & $0.80(0.56-1.15)$ & .300 \\
\hline \multicolumn{6}{|l|}{ Cardiorespiratory fitness } \\
\hline $\mathrm{n}$ & 947 & 940 & 959 & 946 & \\
\hline Man-years of follow-up & 14,440 & 14,633 & 15,048 & 14,968 & \\
\hline No. of cases & 118 & 62 & 47 & 13 & \\
\hline Age-adjusted RR (95\% CI) & 1.00 & $0.54(0.40-0.73)$ & $0.41(0.29-0.58)$ & $0.12(0.07-0.22)$ & $<.001$ \\
\hline Multivariate RR* $(95 \% \mathrm{CI})$ & 1.00 & $0.77(0.56-1.06)$ & $0.71(0.50-1.01)$ & $0.26(0.14-0.47)$ & $<.001$ \\
\hline Multivariate RR§ (95\% CI) & 1.00 & $0.79(0.57-1.08)$ & $0.75(0.53-1.08)$ & $0.28(0.15-0.50)$ & $<.001$ \\
\hline
\end{tabular}

Abbreviations: RR, relative risk

* Model 1: Adjusted for age, BMI, diastolic blood pressure, family history of diabetes, smoking status, and alcohol intake.

$\dagger$ Model 2: Adjusted as for model 1 plus cardiorespiratory fitness.

\$ Model 3: Adjusted as for model 2 plus other muscular and performance fitness.

$\S$ Model 4: Adjusted as for model 1 plus muscular and performance fitness index composite score.

cross-tabulated by quartile of muscular performance fitness index composite scores and low and high cardiorespiratory fitness (Figure 1). Men in the low cardiorespiratory fitness group and the lowest MPF group were used as reference. The lowest relative risk $(0.40,95 \%$ CI 0.21-0.76) was observed among men in the high cardiorespiratory fitness group and the highest MPF group.

\section{Discussion}

In this study, we prospectively investigated the relationship between MPF levels and the incidence of type 2 diabetes in Japanese men. Our results show that low MPF was associated with higher risk for the incidence of type 2 diabetes; however, this inverse association was attenuated once cardiorespiratory fitness was included in the model.

In 2007, the American College of Sports Medicine and the American Heart Association published updated recommendations for physical activity and public health. ${ }^{5}$ In this set of recommendations, the committee recommended increasing muscular strength and endurance because current evidence suggests that MPF may contribute to health benefits. This study suggests that the primary fitness factor for the prevention of type 2 diabetes is cardiorespiratory fitness, which is primarily determined by aerobic exercise habits. However, MPF, especially muscle endurance, may contribute to the prevention of 


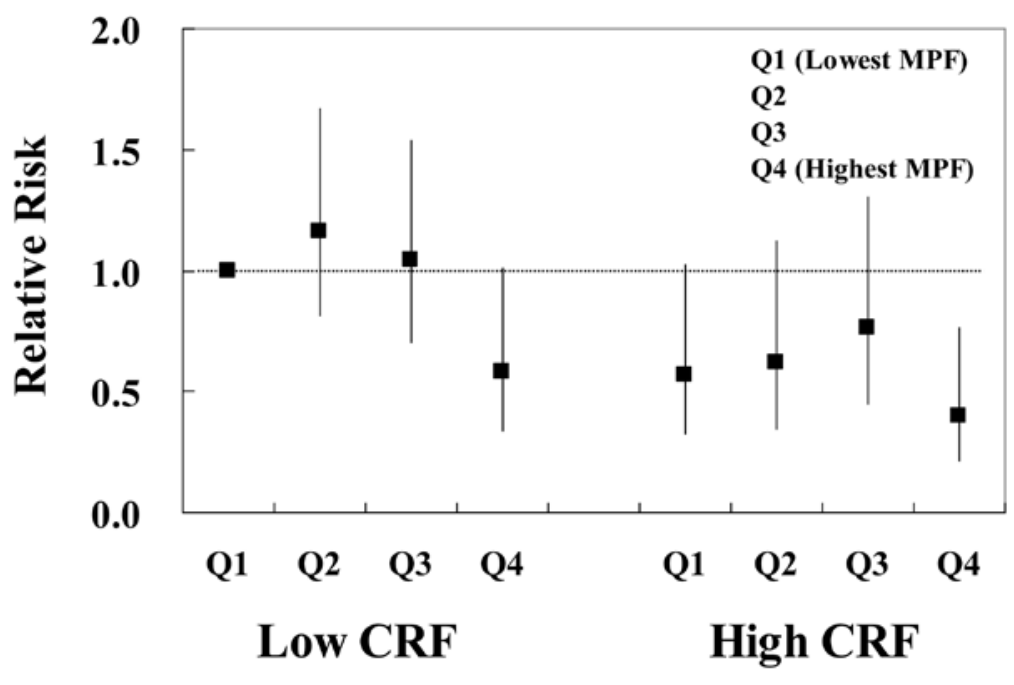

Figure 1 - Relative risks of the incidence of type 2 diabetes, by cardiorespiratory fitness (CRF) and muscular and performance fitness (MPF). Results are adjusted for age, BMI, diastolic blood pressure, family history of diabetes, smoking status, and alcohol intake. Vertical bars indicate $95 \%$ CIs.

type 2 diabetes, particularly among individuals with low cardiorespiratory fitness. Figure 1 shows that among persons with low cardiorespiratory fitness, those with high MPF have a lower relative risk compared with those with low MPF. However, among those with high cardiorespiratory fitness, there was little difference in risk of type 2 diabetes across different MPF groups. To our knowledge, only 1 prospective cohort study has been conducted on the relationship between MPF levels and the incidence of type 2 diabetes. Katzmarzyk et al found a significant inverse relationship between musculoskeletal fitness composite score (calculated from the scores for sit-ups, push-ups, grip strength and trunk flexibility) and the incidence of type 2 diabetes among the Canadian population. ${ }^{9}$ They reported that sit-ups and push-ups, which are both indicators of muscle endurance, were inversely related to risk, but grip strength and trunk flexibility were not. In addition, cardiorespiratory fitness was most strongly associated with risk of type 2 diabetes. Their results are consistent with those from the current study. These findings suggest that endurance (both cardiorespiratory and muscular) is an important fitness component for the prevention of type 2 diabetes.

The major strengths of this study include its prospective design, as well as the objective measurements of different components of MPF. Another advantage of the current study was the screening for type 2 diabetes at the annual clinical examination. Thus, there is no selection bias because over $99.5 \%$ of the subjects completed the examination.

However, there are some limitations to this study. Jurca et al reported that US men with higher muscle strength, measured by leg press and the bench press, have a significantly lower risk of developing metabolic syndrome. ${ }^{14}$ Moreover, several prospective studies suggest that grip strength is a predictor of all-cause mortality among elderly and nonelderly populations. ${ }^{15,16}$ Recently, increasing evidence suggests that muscle strength may contribute to health benefits. ${ }^{17}$ We did not have data on muscle strength; however, we did measure muscular endurance (sit-ups) which is correlated to muscle strength. ${ }^{18}$ In addition, the subjects are not representative of the entire Japanese population, but were men employed in a large metropolitan company.

In conclusion, this prospective study suggests that MPF is a predictor of type 2 diabetes in Japanese men, although its predictive ability was attenuated once cardiorespiratory fitness was accounted for.

\section{Acknowledgments}

We thank the Tokyo Gas Health Promotion Center physicians and staff for assistance with data collection. Also thanks to Robert A. Sloan and Benjamin Howe for helpful comments, and to Ayumi Sawada for secretarial support.

\section{References}

1. Jeon CY, Lokken RP, Hu FB, Van Dam RM. Physical activity of moderate intensity and risk of type 2 diabetes: a systematic review. Diabetes Care. 2007;30:744-752.

2. LaMonte MJ, Blair SN, Church TS. Physical activity and diabetes prevention. J Appl Physiol. 2005;99:1205-1213. 
3. Ivy JL, Zderic TW, Fogt DL. Prevention and treatment of non-insulin-dependent diabetes mellitus. Exerc Sport Sci Rev. 1999;27:1-35.

4. Bouchard C, An P, Rice T, et al. Familial aggregation of VO2max response to exercise training: results from the HERITAGE Family Study. J Appl Physiol. 1999;87:10031008.

5. Haskell WL, Lee IM, Pate RR, et al. Physical activity and public health: updated recommendation for adults from the American College of Sports Medicine and the American Heart Association. Med Sci Sports Exerc. 2007;39:1423-1434.

6. Lynch J, Helmrich SP, Lakka TA, et al. Moderately intense physical activities and high levels of cardiorespiratory fitness reduce the risk of non-insulin-dependent diabetes mellitus in middle-aged men. Arch Intern Med. 1996;156:1307-1314.

7. Sawada SS, Lee IM, Muto T, Matuszaki K, Blair SN. Cardiorespiratory fitness and the incidence of type 2 diabetes: prospective study of Japanese men. Diabetes Care. 2003;26:2918-2922.

8. Wei M, Gibbons LW, Mitchell TL, Kampert JB, Lee CD, Blair SN. The association between cardiorespiratory fitness and impaired fasting glucose and type 2 diabetes mellitus in men. Ann Intern Med. 1999;130:89-96.

9. Katzmarzyk PT, Craig CL, Gauvin L. Adiposity, physical fitness and incident diabetes: the physical activity longitudinal study. Diabetologia. 2007;50:538-544.

10. Åstrand PO, Ryhming I. A nomogram for calculation of aerobic capacity (physical fitness) from pulse rate during sub-maximal work. J Appl Physiol. 1954;7:218-221.
11. Åstrand I. Aerobic work capacity in men and women with special reference to age. Acta Physiol Scand Suppl. 1960;49:1-92.

12. Expert Committee on the Diagnosis and Classification of Diabetes Mellitus. Report of the Expert Committee on the Diagnosis and Classification of Diabetes mellitus (Position Statement). Diabetes Care. 1997;20:1183-1197.

13. Li C, Ford ES, McGuire LC, Mokdad AH. Increasing trends in waist circumference and abdominal obesity among U.S. adults. Obesity (Silver Spring). 2007;15:216223.

14. Jurca R, LaMonte MJ, Church TS, et al. Associations of muscle strength and aerobic fitness with metabolic syndrome in men. Med Sci Sports Exerc. 2004;36:1301-1307.

15. Gale CR, Martyn CN, Cooper C, Sayer AA. Grip strength, body composition, and mortality. Int J Epidemiol. 2007;36:228-235.

16. Sasaki H, Kasagi F, Yamada M, Fujita S. Grip strength predicts cause-specific mortality in middle-aged and elderly persons. Am J Med. 2007;120:337-342.

17. Pollock ML, Franklin BA, Balady GJ, et al. AHA Science Advisory. Resistance exercise in individuals with and without cardiovascular disease: benefits, rationale, safety, and prescription: an advisory from the committee on exercise, rehabilitation, and prevention, council on clinical cardiology, American Heart Association; Position paper endorsed by the American College of Sports Medicine. Circulation. 2000;101:828-833.

18. Katzmarzyk PT, Craig CL. Musculoskeletal fitness and risk of mortality. Med Sci Sports Exerc. 2002;34:740-744. 